Draft Version April 27, 2022

Preprint typeset using $\mathrm{I}_{\mathrm{A}} \mathrm{T}_{\mathrm{E}} \mathrm{X}$ style emulateapj v. 5/2/11

\title{
TIDALLY INDUCED OFFSET DISKS IN MAGELLANIC SPIRAL GALAXIES
}

\author{
Stephen A. PARDy \\ Department of Astronomy, University of Wisconsin, 475 North Charter Street, Madison, WI 53706, USA \\ Elena D'ONGHia ${ }^{1}$ \\ Department of Astronomy, University of Wisconsin, 475 North Charter Street, Madison, WI 53706, USA
}

\author{
E. Athanassoula \\ Aix Marseille Université, CNRS, LAM (Laboratoire d'Astrophysique de Marseille) UMR 7326, 13388, Marseille, France
}

ERIC M. WILCOTS

Department of Astronomy, University of Wisconsin, 475 North Charter Street, Madison, WI 53706, USA

KARTIK SHETH

National Aeronautics and Space Administration Headquarters, 300 E. Street SW, Washington, DC 20546

Draft version April 27, 2022

\begin{abstract}
Magellanic spiral galaxies are a class of one-armed systems that often exhibit an offset stellar bar, and are rarely found around massive spiral galaxies. Using a set of N-body and hydrodynamic simulations we consider a dwarf-dwarf galaxy interaction as the driving mechanism for the formation of this peculiar class of systems. We investigate here the relation between the dynamical, stellar and gaseous disk center and the bar. In all our simulations the bar center always coincides with the dynamical center, while the stellar disk becomes highly asymmetric during the encounter causing the photometric center of the Magellanic galaxy disk to become mismatched with both the bar and the dynamical center. The disk asymmetries persist for almost $2 \mathrm{Gyr}$, the time that it takes for the disk to be re-centered with the bar, and well after the companion has passed. This explains the nature of the offset bar found in many Magellanic-type galaxies, including the Large Magellanic Cloud (LMC) and NGC 3906. In particular, these results, once applied to the LMC, suggest that the dynamical center should reside in the bar center instead of the H I center as previously assumed, pointing to a variation in the current estimate of the north component of the LMC proper-motion.

Subject headings: galaxies: dwarf - galaxies: interactions - galaxies: irregular - galaxies: kinematics and dynamics
\end{abstract}

\section{INTRODUCTION}

Bars are common features in today's Universe. Over two-thirds of all nearby disk galaxies are barred (SaSd) galaxies, including the Milky Way (e.g. de Vaucouleurs 1963; Eskridge et al. 2000; Menendez-Delmestre et al. 2007, Marinova \& Jogee 2007; Sheth et al. 2008). The Spitzer Survey of Stellar Structure in Nearby Galaxies, $\mathrm{S}^{4} \mathrm{G}$, a well-suited survey to identify bars, confirmed these results for the nearby spiral galaxies (Sheth et al. 2010, Muñoz-Mateos et al. 2013, Buta et al. 2015).

However, there is a class of low-mass stellar galaxies, named Magellanic spirals, that show evidence of relatively rare features. In particular, these galaxies are characterized by a bar whose center is displaced from that of the disk, one-armed spirals and an otherwise normal and gas-rich disk (de Vaucouleurs \& Freeman 1972). The Large Magellanic Cloud (LMC) is considered the prototype of this class of objects. However, despite a wealth of data, there is still a good deal of uncertainty concerning the nature of the LMC's bar (see D'Onghia \&

spardy@astro.wisc.edu

${ }^{1}$ Altred P. Sloan Fellow
Fox (2015) for a review on the subject). Work by van der Marel (2001) found that the bar is offset from the dynamical center of the LMC and resides within a large stellar disk. Earlier work by Zhao \& Evans (2000) described the bar as being an unvirialized structure that is offset from the rest of the disk as a result of the LMC's interaction with the Small Magellanic Cloud (SMC). Subramaniam \& Subramanian (2009) used the OGLE III survey (Udalski et al. 2008) and contend that the LMC's bar resides in the plane of the disk. Lastly, there is no evidence of a bar in the H I maps of the LMC presented in Staveley-Smith et al. (2003).

The dynamics, structure, and star formation history of the LMC have long been interpreted in the context of its proximity to both the Milky Way and the SMC. The majority of the observed Magellanic spirals in the nearby Universe share the LMC's structure, in particular the evidence of an offset bar and a one-armed spiral structure, but are rarely found around massive spirals (Wilcots \& Prescott 2004). A good example of these systems is offered by NGC 3906 that shows evidence of the bar offset from the photometric center of the galaxy by $1.2 \mathrm{kpc}$ (see, e.g., Figure 1 of de Swardt et al. 2015), or NGC 
4618 (Odewahn 1991). As more galaxies have been examined from the $\mathrm{S}^{4} \mathrm{G}$ survey (Sheth et al. 2010), it has been found that these offset bars, though rare, represent as many as $5 \%$ of all barred spirals (Ross 2012, Sheth, K. et al. 2016, in preparation).

The dynamics of Magellanic barred spirals is much more complex than that of standard galaxies with centered bars, as could be expected since the different components, disk and bar, have different centers. de Vaucouleurs \& Freeman (1972) studied the orbital structure of such galaxies using an axisymmetric and a bar potential whose centers do not coincide. Colin \& Athanassoula (1989) calculated the corresponding shifts of the Lagrangian points, as well as the response density and velocity fields in such potentials. They found that the center of the velocity field does not necessarily coincide either with the center of the bar or with that of the axisymmetric component, but may be at an intermediate position, its specific location depending on the geometry and size of the offset.

N-body simulations allowed for self-consistent modeling and the introduction of a perturbing companion. Athanassoula (1996) reproduced the one-armed morphology and noted the importance of the impact position with respect to the bar, while Bekki (2009) discussed whether the offset observed in the LMC could be due to a 'dark' companion. Athanassoula et al. (1997) found that the displacements of the centers are accompanied by changes of the bar pattern speed and size. This work was extended by Berentzen et al. (2003) who introduced gas in the simulation and found that it is possible to destroy the bar while keeping the disc structure. More recently, numerical experiments suggest a dwarf-dwarf galaxy interaction origin for the offset bar and one-arm structure (Besla et al. 2012; Yozin \& Bekki 2014). The tidal interactions between dwarf galaxies with different masses play a key role in the stellar and gas stripping in low-mass systems as a consequence of resonant interactions between spinning discs (D'Onghia et al. 2009, 2010, Eokas et al. 2015. Gajda \& Lokas 2015) and explain the morphology and origin of the Magellanic Stream (Besla et al. 2010. 2012).

In this study we aim to understand the structure and dynamics of Magellanic spirals with similar characteristics to the LMC. In particular, we explore whether direct dwarf-dwarf collisions with a mass ratio of 1:10 produce the asymmetric structures characteristic of Magellanictype galaxies and can account for the internal morphology and kinematics of this general class of galaxies. While previous works showed that these systems can have offset bars originated by encounters with a less massive companion, here we show that the bar is never off-centered. Instead, the stellar disk is shifted from the dynamical center as a consequence of the impact with a companion galaxy.

The paper is organized as follows. In Section 2, we outline our methodology and describe numerical models involving the direct collision between a Magellanic galaxy and a companion. We then analyze the outcome of the numerical experiments in Section 3. A comparison of the models and the observations is also presented in Section 3.4. We then discuss the implications to the estimate of the LMC proper-motion and conclude with a summary.

\section{NUMERICAL METHODS}

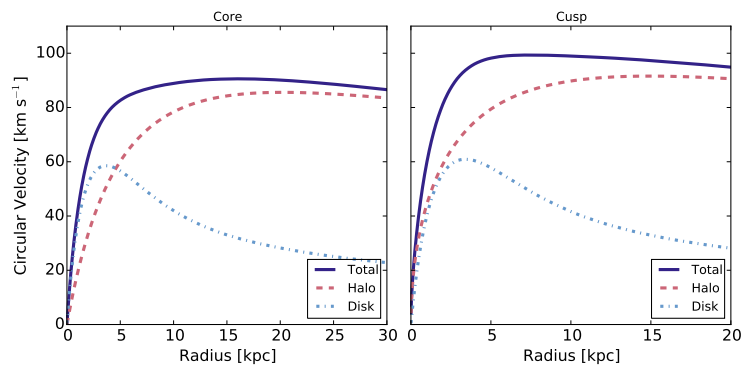

Figure 1. Rotational curves for the primary galaxy when a cored dark profile (left panel) or Hernquist (right panel) is adopted. Both galaxies have the same total mass. A thin exponential disk for both the stars and gas is assumed, with the combined profile shown (light-blue dot-dash line).

We carried out a set of simulations with GADGET3, a parallel TreePM Smoothed particle hydrodynamics (SPH) code developed to compute the evolution of stars and dark matter, which are treated as collisionless fluids and gas. A detailed description of the code is available in the literature (Springel 2005). GADGET3 computes the short-range forces using a tree-based hierarchical multipole expansion. Pairwise particle interactions are softened with a spline kernel of scale-length $h_{s}$, so that they are strictly Newtonian for particles separated by more than $h_{s}$. The resulting force is roughly equivalent to traditional Plummer softening with scale length $h \approx h_{s} / 2.8$. For our applications the gravitational softening length is fixed to $h_{s}=120 \mathrm{pc}$ throughout the evolution of the galaxy encounters.

The GADGET3 code incorporates a subresolution multiphase model of the interstellar medium (ISM) including radiative cooling (Springel \& Hernquist 2003) and a fully conservative approach to integrating the equations of motion (Springel \& Hernquist 2002). The simulations presented in this work include gas physics but are not aimed at describing properties of star formation or feedback.

\subsection{Initial Conditions}

The setup of each galaxy in our study consists of a dark matter halo and a rotationally supported disk of stars and gas. The parameters describing each component are independent, and models are chosen with orbital parameters as described below.

Each disk galaxy was generated using the GalIC code described in Yurin \& Springel (2014), which uses an iterative approach for the realization of equilibrium N-body systems for given density distributions (Rodionov et al. 2009). This is achieved for arbitrary axisymmetric density structure and by taking the mutual influence of different mass components fully into account.

The initial conditions for the setup of a Magellanictype galaxy and a companion galaxy used for all models are summarized in Table 1 . The total mass for the Magellanic spiral is assumed to be an LMC analog, with parameters with values as in Besla et al. (2012). The companion galaxy is then chosen to be 10 times less massive than the primary galaxy. 
The number of particles of each component (gas, stars, dark matter) is chosen such that the mass resolution per particle of a given type is roughly the same in both galaxies. The primary Magellanic-type galaxy is modeled with gas and stellar disks with the same scale length, with the values indicated in Table 1

Table 1

Structural Parameters of the Primary and Companion Galaxy

\begin{tabular}{|c|c|c|c|}
\hline \multirow[b]{2}{*}{ Parameters } & \multicolumn{2}{|c|}{ Primary Galaxy } & \multirow[t]{2}{*}{ Companion } \\
\hline & Cored Halo & Hernquist Halo & \\
\hline $\mathrm{a}(\mathrm{kpc})^{\mathrm{a}}$ & 11.3 & 14.9 & 5.8 \\
\hline $\mathrm{r}_{d}(\mathrm{kpc}) \mathrm{b}$ & 1.6 & 1.6 & 0.6 \\
\hline $\mathrm{M}_{D M}\left(\times 10^{10} \mathrm{M}_{\odot}\right)^{\mathrm{c}}$ & 12 & 12 & 1.1025 \\
\hline $\mathrm{M}_{\text {disk }}\left(\times 10^{10} \mathrm{M}_{\odot}\right)^{\mathrm{d}}$ & 2.52 & 2.52 & 0.055 \\
\hline $\mathrm{M}_{\text {gas }}\left(\times 10^{10} \mathrm{M}_{\odot}\right)^{\mathrm{e}}$ & 1.08 & 1.08 & $\cdots$ \\
\hline $\mathrm{f}_{\text {gas }}^{\mathrm{f}}$ & 0.3 & 0.3 & \\
\hline $\mathrm{N}_{\text {halo }}^{\mathrm{g}}$ & $2 \times 10^{6}$ & $2 \times 10^{6}$ & $3.61 \times 10^{5}$ \\
\hline $\mathrm{N}_{\text {disk }}{ }^{\mathrm{h}}$ & $7 \times 10^{5}$ & $7 \times 10^{5}$ & $3 \times 10^{5}$ \\
\hline $\mathrm{N}_{\text {gas }}{ }^{\mathrm{i}}$ & $3 \times 10^{5}$ & $3 \times 10^{5}$ & $\cdots$ \\
\hline
\end{tabular}

The disk component in each interacting galaxy has a thin exponential surface density profile of scale length $r_{d}$ :

$$
\Sigma_{d i s k}=\frac{M_{d i s k}}{2 \pi r_{d}^{2}} \exp \left(-r / r_{d}\right),
$$

so that the disk mass is $\mathrm{M}_{d i s k}=m_{d} \mathrm{M}_{\mathrm{DM}}$, where $m_{d}$ is dimensionless and $\mathrm{M}_{\mathrm{DM}}$ is the total halo mass. The vertical mass distribution of the stars in the disk is specified by giving it the profile of an isothermal sheet with a radially constant scale height $z_{0}$. The $3 \mathrm{D}$ stellar density in the of stars and gas disk is hence given by:

$$
\rho_{*}(r, z)=\frac{M_{\text {disk }}}{4 \pi z_{0} r_{d}^{2}} \operatorname{sech}^{2}\left(\frac{\mathrm{z}}{\mathrm{z}_{0}}\right) \exp \left(-\frac{\mathrm{r}}{\mathrm{r}_{\mathrm{d}}}\right)
$$

The scale height of the stellar disk is adopted as 0.2 of the disk scale length. The gaseous disk height is initially set equal to the stellar disk height. The energy and pressure of the ISM are prescribed by the chosen effective equation of state (Springel et al. 2005).

The models for the primary Magellanic-type galaxy assume a dark halo with the following general form (Dehnen 1993):

$$
\rho(r)=\frac{(3-\gamma) M_{D M}}{4 \pi} \frac{a}{r^{\gamma}(r+a)^{4-\gamma}},
$$

where $M_{\mathrm{DM}}$ is the galaxy halo mass, $a$ is the scale length of the halo, and $\gamma$ is a parameter that determines the shape of the profile. For $\gamma=1$ the halo has a central cusp and follows a Hernquist model (Hernquist 1990), while for $\gamma=0$ the density profile belongs to the same family of density profiles but with a constant-density core. The isotropic distribution function for the energy is given in this case by (Dehnen 1993):

$f(\epsilon)=\frac{(3-\gamma) M}{2\left(2 \pi^{2} G M a\right)^{3 / 2}} \int_{0}^{\epsilon} \frac{(1-y)^{2}\left[\gamma+2 y+(4-\gamma) y^{2}\right]}{y^{4-\gamma} \sqrt{\epsilon-\Psi}} d \Psi$

where $\epsilon=-E(G M / a)^{-1}$ is the dimensionless binding energy.

The total galaxy mass and disk mass fraction for the primary galaxy are fixed to be the same in both models. Additionally, the scale lengths for the cored and Hernquist halo models are set such that both galaxies have similar rotation velocity $V_{\text {tot }}$ at the radius that enclosed the total mass of the galaxy.

Figure 1 displays the resulting rotation curves for the primary Magellanic-type galaxy when a cored density profile (left panel) or a Hernquist profile (right panel) is adopted. The initial total rotation curve of the primary galaxy peaks at $V_{\text {rot }}=90 \mathrm{~km} \mathrm{~s}^{-1}$ at two halo scale lengths from the center in the cored profile, whereas the peak is $100 \mathrm{~km} \mathrm{~s}^{-1}$ for the cusped Hernquist model at one halo scale length. This gives a total mass within $8 \mathrm{kpc}$ for the cored (cusped) galaxy of $1.35(1.77) \times 10^{10} \mathrm{M}_{\odot}$. Note that the disk fraction within $2 r_{d}$ is higher in the cored galaxy with respect to the Hernquist galaxy.

This study focuses on the response of the disk of a Magellanic-type galaxy when perturbed by a close encounter with a lower-mass companion. The initial stellar disks of the Magellanic-type galaxies are chosen to be sufficiently bar unstable for the bar to grow fast in isolation (D'Onghia 2015); hence, the bar feature in all models has not been induced by external tidal perturbations induced by the companion (Eokas et al. 2014), but grew from the beginning of the simulation.

The perturber is modeled assuming an exponential stellar disk and a Hernquist dark matter density profile, with the choice of parameters listed in Table 1.

\subsection{Orbital configurations}

Our set of simulations presents four orbital configurations for each halo model. In each set of simulations we let the primary galaxy evolve over a period of $4 \mathrm{Gyr}$ in order to grow a stellar bar at the center and to settle to equilibrium before we introduced a companion. At the start of the simulation, the companion was placed at $\sim$ $50 \mathrm{kpc}$ on a fast single passage orbit. Visual inspection of the primary disk after the companion has been added shows no perturbations until the impact occurs. The initial position and velocities of the companion galaxy in the set of simulations are presented in Table 2. The values are assumed in the coordinate system at the center of mass of the primary galaxy. The initial orbital position and velocity of the interacting galaxies are set so that all encounters had an impact parameter of $4 \pm 0.06 \mathrm{kpc}$ and a relative velocity of $337 \pm 4 \mathrm{~km} \mathrm{~s}^{-1}$. This value is motivated by simulations of the LMC in which an offset bar is created by a direct impact by the SMC (Besla et al. 2012).

Two out of four orbital configurations are coplanar $(\theta$ $=0)$. One is a prograde, and the other is retrograde. The other two orbits are both prograde and inclined with respect to the plane of the primary galaxy by $45^{\circ}$ and $90^{\circ}$, respectively. The encounter occurred at $\sim 0.15 \mathrm{Gyr}$ and the primary galaxy was followed for 4 Gyr after the 


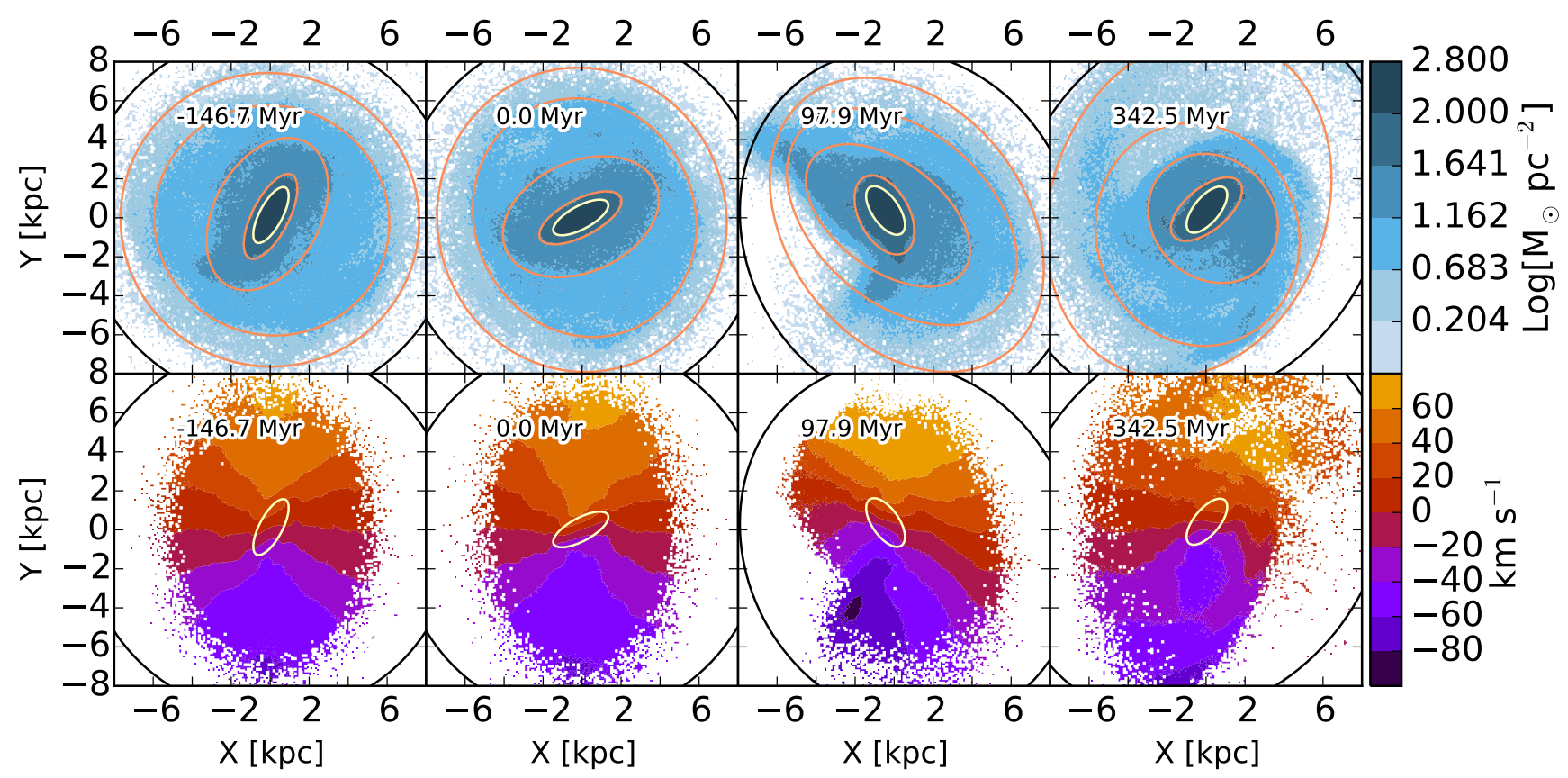

Figure 2. Time sequence of the stellar and gaseous disks of the primary galaxy. The leftmost panel corresponds to the time immediately before the companion is introduced. Time $0(t=0)$ is set at the time of the closest approach with the perturber. The primary galaxy is modeled here with a cored halo density model. Note that the stellar and gas distributions begin orderly but become disturbed during the encounter with the companion. The top row shows the face-on stellar disk surface density map. Isodensity ellipses (orange lines) are superimposed. The inner ellipse is used to identify the bar (yellow), while the outer ellipse outlines the disk (black). The encounter in this model is prograde with an inclination of $45^{\circ}$. The companion is not displayed in the image sequence. The bottom row displays the time evolution of the gas velocity fields of the same galaxy. The effect of the bar is apparent in the first panel as an asymmetry in the inner contours.

Table 2

ORBITAL PARAMETERS OF NUMERICAL MODELS.

\begin{tabular}{lcccccr}
\hline Model & $\mathrm{x}$ & $\begin{array}{c}\mathrm{y} \\
(\mathrm{kpc})\end{array}$ & $\mathrm{z}$ & $\mathrm{Vx}$ & $\mathrm{Vy}$ \\
$\left(\mathrm{km} \mathrm{s}^{-1}\right)$ & $\mathrm{Vz}$ \\
\hline Cored, $\theta=45^{\circ}$ & 29 & 36 & 29 & -147 & -147 & -147 \\
Cored, $\theta=90^{\circ}$ & 0 & 6 & 50 & 0 & 0 & -255 \\
Cored, $\theta=0^{\circ}$ & 50 & 7 & 0 & -260 & 0 & 0 \\
Cored, $\theta=0^{\circ}$ Retrograde & 50 & 7 & 0 & -265 & 0 & 0 \\
Hernquist, $\theta=45^{\circ}$ & 29 & 35 & 29 & -147 & -147 & -147 \\
Hernquist, $\theta=90^{\circ}$ & 0 & 3.75 & 50 & 0 & 0 & -259 \\
Hernquist, $\theta=0^{\circ}$ & 50 & 3.5 & 0 & -255 & 0 & 0 \\
Hernquist, $\theta=0^{\circ}$ Retrograde & 50 & 3.5 & 0 & -255 & 0 & 0 \\
\hline
\end{tabular}

Note: The origin of the coordinate system is at the center-of-mass of the primary galaxy.

simulation started.

\subsection{Quantifying disk off-centers and lopsidedness}

In order to quantify the bar displacement in the Magellanic-type galaxy in tidal interaction with a companion galaxy, we first need to identify the centers of the galaxy components: stellar and gas disks and the stellar bar. An observationally motivated approach has been used to define disk and bar centers as in the study of the Magellanic spiral NGC 3906 by de Swardt et al. (2015).

The stellar or gaseous disk of the primary galaxy is projected to a face-on density map with 256 square bins in each dimension. Each bin has a size of $\sim 78 \mathrm{pc}$, corresponding to $\sim 1$ arcsecond resolution at the distance of the observed galaxy NGC 3906. We varied the bin size in the analysis and found the effect to be minimal. The density map is then cut into logarithmically spaced intensity contours. Each contour is fit with ellipses. The outer ellipse serves the stellar disk, while the innermost ellipse with eccentricity greater than 0.5 outlines the bar (Yozin \& Bekki 2014). Various methods have been used to identify the bar in our simulations; however, this approach produces the best fits to visual inspection, and allows for comparisons with previous analyses. The bar center has also been measured adopting the brightest pixel following the suggestion of van der Marel \& Kallivayalil (2014), and we found that the bar center estimated in this way agrees with the values obtained with the ellipse method, with the uncertainties estimated to be within the numerical resolution.

To find the disk and bar centers, we fit ellipses to the isodensity contours of our primary galaxy. The disk center is probed by our outermost ellipse fit, which we set at a density of $0.5 \mathrm{M}_{\odot} p c^{-2}$. The ellipse that fits this outer isodensity contour has a typical radius of $10 \mathrm{kpc}$. We tested the effect of measuring the offset from the dynamical center using a higher density cutoff of $10.7 \mathrm{M}_{\odot} p c^{-2}$. This density probes an inner disk with typical radii of 5 kpc. The inner disk shows similar qualitative results and a similar time evolution, but with reduced amplitude.

The galaxy dynamical center is defined as the location where the galaxy potential well is deepest. We measure it by taking the center of mass of the 100 particles with the most negative potentials. We also measured the center of the dark matter alone by repeating the same procedure, but using only dark matter particles. Throughout the paper the bar and disk offsets refer to the displacement of that component with the galaxy dynamical center, but the implications of using the halo center instead are minimal and will be briefly discussed in Section 3.1 The in-plane separation between the disk and bar centers is used as a measure of the bar-disk offset (what is 
traditionally called the "offset bar").

An example of the photometric fitting approach applied to the stellar primary galaxy during the gravitational encounter with a companion is illustrated in Figure 2 (top row), which shows the projected surface density and isodensity ellipses superimposed (orange lines). The inner ellipse (yellow line) identifies the stellar bar, and the outer ellipse (black line) fit the outer stellar disk. The panels are labeled by time, with the initial time of the simulation set to the time when the companion is first introduced and $t=0$ as the time when the perturber hits the disk of the primary galaxy.

\subsection{Quantifying the disk lopsidedness}

In our simulations the asymmetries of the stellar disk and the lopsidedness are determined by projecting the disk face-on and measuring its stellar surface density, $\Sigma(r, \phi)$, with $(r, \phi)$ being polar coordinates in the disk plane. If the surface brightness distribution is invariant for a rotation of $2 \pi / m$ rad, so that $\Sigma(r, \phi+2 \pi / m)=$ $\Sigma(r, \phi)$, then the galaxy has $m$-fold rotational symmetry and has $m$ arms and a bar for $m=2$.

If the surface brightness is expressed as a Fourier series, then the disk lopsidedness, the strength of the bar, and the amplitude of the spiral structure can be measured from its Fourier components, which are calculated as

$$
\begin{array}{r}
\Sigma_{m c}(r)=2\langle\Sigma(r, \phi) \cos m \phi\rangle \\
\Sigma_{m s}(r)=2\langle\Sigma(r, \phi) \sin m \phi\rangle \\
\text { for } \mathrm{m}=1,2, \ldots, \infty \\
\Sigma_{0}=2\langle\Sigma(r, \phi)\rangle
\end{array}
$$

The amplitude of each Fourier component is calculated relative to $\Sigma_{0}$ and can take values between 0 and 1:

$$
A_{m}=\frac{\sqrt{\Sigma_{m c}^{2}+\Sigma_{m s}^{2}}}{\Sigma_{0}} .
$$

The face-on disk is divided into concentric annuli, which are further divided into azimuthal bins. The mass from the star particles is then assigned to these bins, making it possible to compute the stellar surface density $\Sigma(r, \phi)$. Fourier components of the surface density are then calculated according to Equation 5 .

Following Zaritsky \& Rix (1997) and Zaritsky et al. (2013), we then measure the average of the Fourier components' amplitudes $\left\langle A_{m}\right\rangle$ (for $m=1$ and $m=2$ ) between 1.5 and 2.5 scale lengths $(2.2-3.7 \mathrm{kpc}$ for the disk scale length of $1.4 \mathrm{kpc})$. We repeat this measurement during the time of the interaction between the primary galaxy and the companion to track the growth of asymmetries and the strength of the bar.

\subsection{Gas disk velocity fields}

A weighted two-dimensional velocity map for the gas is inferred in our simulations projecting the galaxy as if it had been observed at a certain inclination and then taking the mass-weighted average of the radial velocity components of the gas particles. An example of twodimensional velocity maps is illustrated in the bottom row of Figure 2, where the galaxy is observed with an inclination of $45^{\circ}$ starting before the companion is introduced and following several gigayears of evolution.

\section{RESULTS}

\subsection{Stellar disk dynamical response}

We investigate perturbations induced in the stellar disk of the primary galaxy by a recent encounter with a companion exploring four different orbital configurations: a coplanar (prograde and retrograde) an inclined orbit of $45^{\circ}$ and $90^{\circ}$, respectively.

Figure 2 shows the live disk displayed face-on during the $45^{\circ}$ direct encounter with the companion. In this numerical experiment the disk galaxy is embedded in a cored dark matter halo. There are observed asymmetries in the mass distribution of the stellar disk noticeable in the outer parts, which appear shortly after the encounter.

Next, we measured the dynamical center, the bar, and the disk photometric center. Concentric ellipses are superimposed to the disk surface density as shown in Figure 2, The inner ellipse-marked in yellow-outlines the bar, and the ellipse colored in black matches the outer disk density. The time of closest approach happens roughly 0.15 Gyr from the beginning of the simulation. The companion galaxy passes through the primary galaxy quickly $(\sim 0.1$ Gyr within $20 \mathrm{kpc}$ of the primary galaxy) and is within the virial radius of the primary galaxy for $<0.5$ Gyr.

We repeated the experiment with different sets of models varying the orbital inclination angle. We measure the displacements between the dynamical center, the stellar disk, and the bar. Figure 3 quantifies the displacement of the bar and the stellar disk from the dynamical center and displays it as a function of time for encounters run with different orbital inclinations. We also ran our experiments on the isolated disks to measure the intrinsic scatter. In general, this scatter is quite low and well behaved.

After the encounter with the companion (labeled as $t=$ 0 ), the stellar disk responds to the gravitational perturbation by becoming asymmetric in its mass distribution (see the third panel in Figure 2). We note first that the measured bar center is always coincident with the dynamical center. Hence, there is no evidence of off-center bars for any orbital configuration. The stellar disk, however, is measured to be $1.5-2.5 \mathrm{kpc}$ shifted from the dynamic center of each primary galaxy. This readjustment of the disk occurs over a period of approximately 2 Gyr, with small offsets of $\sim 0.5 \mathrm{kpc}$ persisting for another 2 Gyr. During this time, the disk center will be displaced with respect to the bar center up to $2.5 \mathrm{kpc}$ for encounters with orbital inclination angles of $90^{\circ}$ (the largest of the observed shifts).

The displacement of the primary galaxy's stellar disk is caused by the companion galaxy passing though the disk. This fly-by creates strong asymmetries by scattering stars to large radii on one side of the galaxy. This effect is more severe when the impact occurs in the $90^{\circ}$ orbit, producing the strongest disk response and largest displacements from the dynamical center. In some cases, the asymmetric material appears as a single spiral arm.

The disk distortions and asymmetries persist for almost 2 Gyr, the time that it takes for the disk to be recentered, and well after the perturber has passed. The response of the stellar disk appears to oscillate with time as it reduces amplitude, as also noted in Yozin \& Bekki (2014). The primary offset is the most promi- 


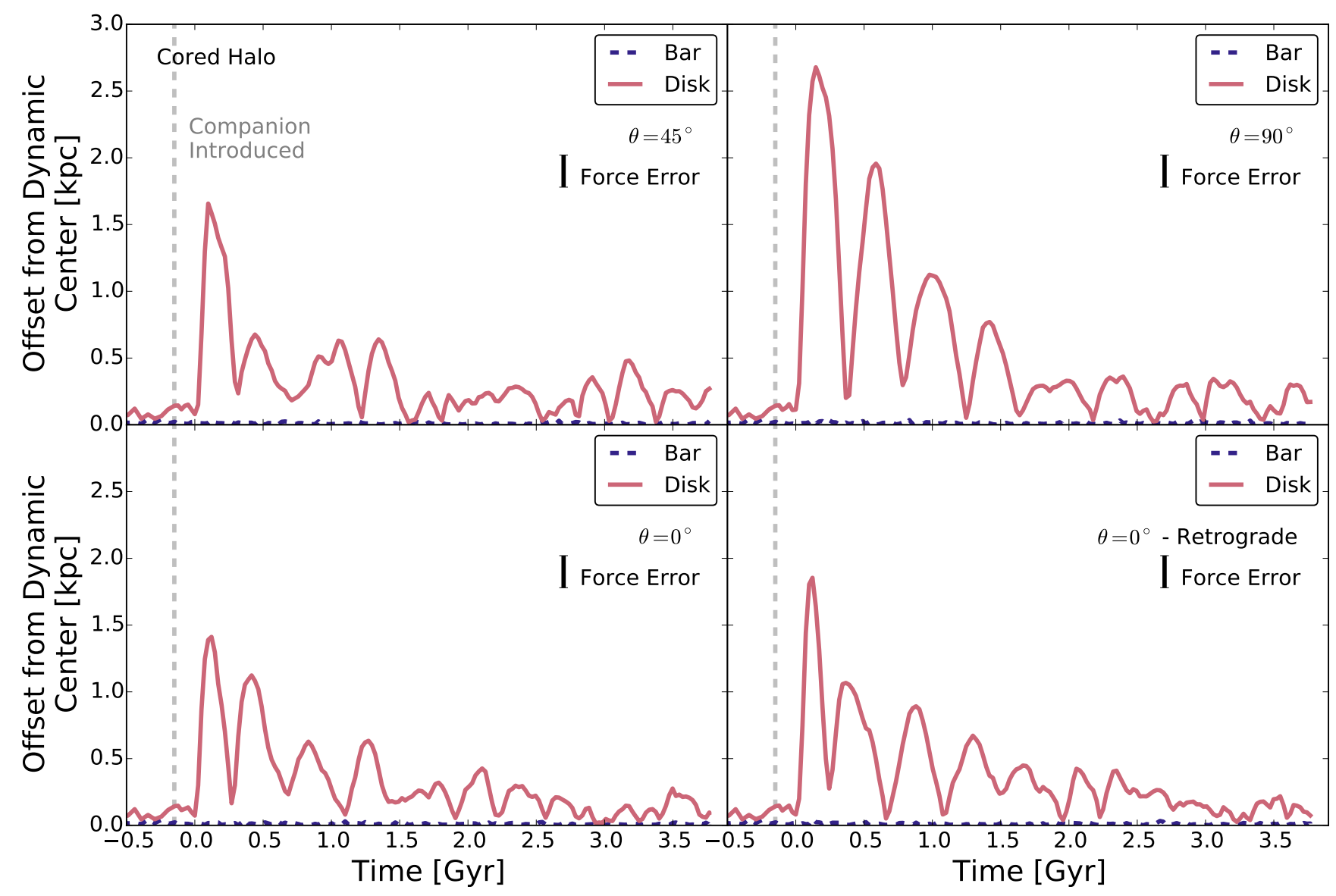

Figure 3. Time evolution of offsets between the photometric center of the disk (solid red line) and bar (dotted blue line) and the dynamical center in the cored halo model for different orbital inclination angles. The size of the error bar is twice the typical force softening length $h_{s}=120 \mathrm{pc}$. The time of closest approach is set at $0 \mathrm{Gyr}$ and we include $0.5 \mathrm{Gyr}$ of evolution before the introduction of a companion. The gray vertical line indicates when the companion was introduced. Top left: $\theta=45^{\circ}$. Top right: $\theta=90^{\circ}$. Bottom left: $\theta=0^{\circ}$. Bottom right: $\theta=0^{\circ}$ (retrograde collision).

nent. The subsequent offsets have amplitudes that decrease strongly with the time since the interaction and soon reach values close to our spatial resolution limits and do not appear to be correlated with disk rotation or other disk properties.

We also tested the offset using the center of the dark matter halo instead of the dynamical center. The dark matter halo center is aligned with the dynamical center before the interaction but becomes offset from both the dynamical center and the bar during the passage of the companion. After the interaction ends, the halo and dynamical centers coincide again and align with the bar.

During testing of our orbital configurations, we made runs with various impact parameters. We took care to hold the impact parameter constant across all simulations with different configurations. Decreasing the impact parameter increased the observed bar-disk offset and measured asymmetries.

As a final test of these results, we experimented with fitting an ellipse to a higher-density region of the disk. This ellipse probes the inner disk of the primary galaxy (defined by a density cutoff of $10.7 \mathrm{M}_{\odot} p c^{-2}$ ), with a typical radius of $5 \mathrm{kpc}$ (vs. our outer disk cutoff of 0.5 $\mathrm{M}_{\odot} p c^{-2}$, which has a typical radius of $10 \mathrm{kpc}$ ). As an example in Figure 4, we show only the most different result between the inner and outer disk which occurs for the $\theta=90^{\circ}$ orbit in the cored halo. Although the qualitative results are the same, with an offset directly after the encounter followed by a gradual damping, the amplitude of the effect is strongly reduced when using the center of the inner disk.

\subsection{Dependence on the shape of halo density profile}

There is some evidence that dwarf disk galaxies do show slowly rising rotation curves that would be consistent with having underlying halos with shallow innerdensity profiles (de Blok et al., 2001, Oh et al. 2011; McGaugh, 2002 but see also Oman et al., 2015). Indeed, if Magellanic disk galaxies are embedded in halos with less concentrated mass distributions in the inner regions, then we may expect an enhancement in the amplitude of the disk oscillations around the dynamical center. If offcentered bars are a real phenomenon, then it is plausible to posit that these oscillations would cause the bar to remain more offset, and for longer, than in a galaxy with a cosmological dark matter halo. To test whether this was driving the offsets seen in the previous section, we also set up models for the Magellanic disk galaxy with a cusped Hernquist dark matter density profile in the inner parts.

Figure 5 illustrates the outcome. Different panels display the time evolution of the disk and bar offset for encounters (the in-plane separation between the mea- 


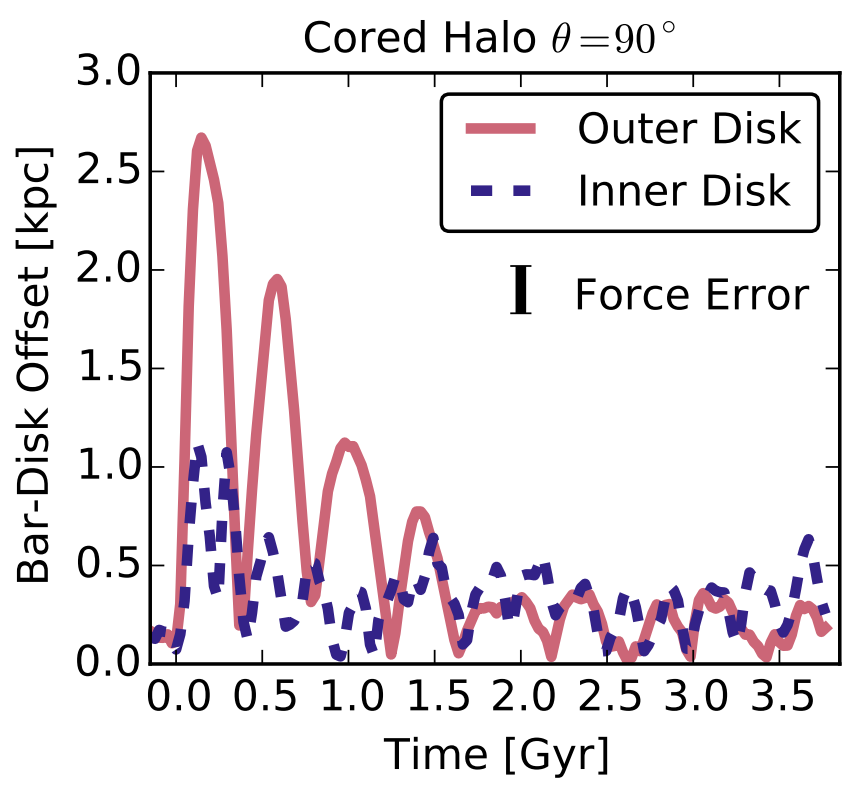

Figure 4. Time evolution of the displacement between the bar and disk centers shown for the $\theta=90^{\circ}$ orbit in the cored halo. We show the effect of using a higher density cutoff which probes the inner disk, with a typical radius of $5 \mathrm{kpc}$. The inner disk cutoff is $10.7 \mathrm{M}_{\odot} p c^{-2}$ while the outer disk cutoff is $0.5 \mathrm{M}_{\odot} p c^{-2}$. This example is the most extreme difference of any of our simulations, but still shows a strong offset between the measured disk and bar centers directly after impact.

sured bar and disk centers) with different orbital configurations. The largest differences are seen in the $90^{\circ}$ orbit, where the maximum (average) displacement is 0.6 (2.7) $\mathrm{kpc}$ for the cored profile, compared to 0.5 (2.1) kpc for the cusped profile. The separation measured for the co-planar retrograde $0^{\circ}$ case consists in the maximum offset of 0.4 (1.9) kpc for the cored profile, as compared to the displacement of $0.3(1.7) \mathrm{kpc}$ measured for the cusped profile.

The $45^{\circ}$ inclined orbit shows a slightly stronger offset for the model with the cusped halo, but this difference and the difference seen in the co-planar prograde $\left(\theta=0^{\circ}\right)$ orbit are both close to the spatial resolution of our models. These results support the conclusion that the bardisk offsets are driven by asymmetries in the outer part of the disk.

\subsection{Stellar disk lopsidedness}

If offsets are the results of an asymmetric disk in the outer parts, then we expect this to appear in the Fourier components analysis. To test this the Fourier components of the stellar disk of the primary galaxy have been measured as a function of time, during the interaction with the companion and long after the encounter ended. Our galaxies begin with strong $A_{2}$ amplitudes indicating symmetric stellar features, and low $A_{1}$ amplitudes.

The outcome is displayed in Figure 6 for the primary galaxy with a cored halo. The results for the Hernquist halo are qualitatively similar. The four panels show the $A_{1}$ and bar-disk offset normalized to their maximum value in the four orbital configurations.

We find that $A_{1}$ becomes pronounced during the peaks of the observed bar-disk offset. These peaks line up remarkably well for $\sim 2$ Gyr after the encounter, at which point the offsets subside. We can quantify the degree to which the two time series coincide by measuring the $\mathrm{R}^{2}$ coefficient:

$$
R^{2}=1-\frac{\sum_{t=0}^{4}(o(t)-\overline{o(t)})^{2}}{\sum_{t=0}^{4}(o(t)-f(t))^{2}},
$$

where, in this case, $o(t)$ is the measured bar-disk offset at time $t$ normalized to its maximum value and $f(t)$ is the $A_{1}$ fourier amplitude at time $t$ normalized to its maximum value. An $\mathrm{R}^{2}$ value of 1 would indicate that the value of the $A_{1}$ mode can explain $100 \%$ of the bar-disk offsets, while an $\mathrm{R}^{2}$ value of 0 would indicate that the the $A_{1}$ mode cannot explain any of the bar-disk offsets. It is important to note, however, that this value merely describes the similarity of the two datasets and cannot prescribe any physical causation one way or the other. The lowest $\mathrm{R}^{2}$ coefficient is found for the $\theta=45^{\circ}$ configuration with $\mathrm{R}^{2}=0.68$. The highest value is found for the $\theta=90^{\circ}$ with $\mathrm{R}^{2}=0.94$.

In every orbital configuration modeled except when $\theta=0^{\circ}$ in a retrograde orbit, $A_{1}$ becomes the dominant mode directly after encounter and remains elevated from its initial amplitude for the entire 4 Gyr. For the $\theta=0^{\circ}$ retrograde orbit $A_{1}$ is dominant for a short period of time, and settles within 3 Gyr. Using the criteria of Bournaud et al. (2005) and Mapelli et al. (2008) $\left(\mathrm{A}_{1} \geq 0.05\right)$, the galaxies showing evidence for bar-disk offsets all appear asymmetric during these periods, a result also found in Athanassoula et al. (1997) and Berentzen et al. (2003). The average bar amplitude in our cored halo shows a modest decrease after the interaction.

Although qualitatively similar (both show higher $A_{2}$ amplitudes before the interaction with the companion and strong $A_{1}$ amplitudes during the tidal interaction), the results for the Hernquist halo have a number of notable differences. The Hernquist Fourier component is $\mathrm{A}_{2}<0.2$ before interaction, even though the galaxy appears strongly barred according to our visual inspection. During the tidal interaction, both $A_{1}$ and $A_{2}$ dominate over the higher Fourier components.

Because of this, we can conclude that the shift of the disk center away from the dynamical center is due to the extended tidal material created after the interaction with the smaller companion. The method of measuring disk centers using fits to isodensity contours appears to be sensitive to larger asymmetric features on the edge of the disk. This conclusion is further supported by the fact that the typical radius measured by the outer isodensity contours increases during the encounter with the companion.

\subsection{Gas disk dynamical response}

In the simulations described in the previous sections, we showed that the bar accurately reflects the dynamical center, while the stellar disk exhibits a prominent offset. Wilcots \& Prescott (2004), however, find that the majority of the observed Magellanic galaxies have H I profiles no more or less asymmetric than other late type spirals found in Matthews \& Gallagher (2002). To test the effects of the gravitational encounter with a companion on the gas distribution of the primary galaxy, we measured the centers of the simulated gas velocity fields adopt- 


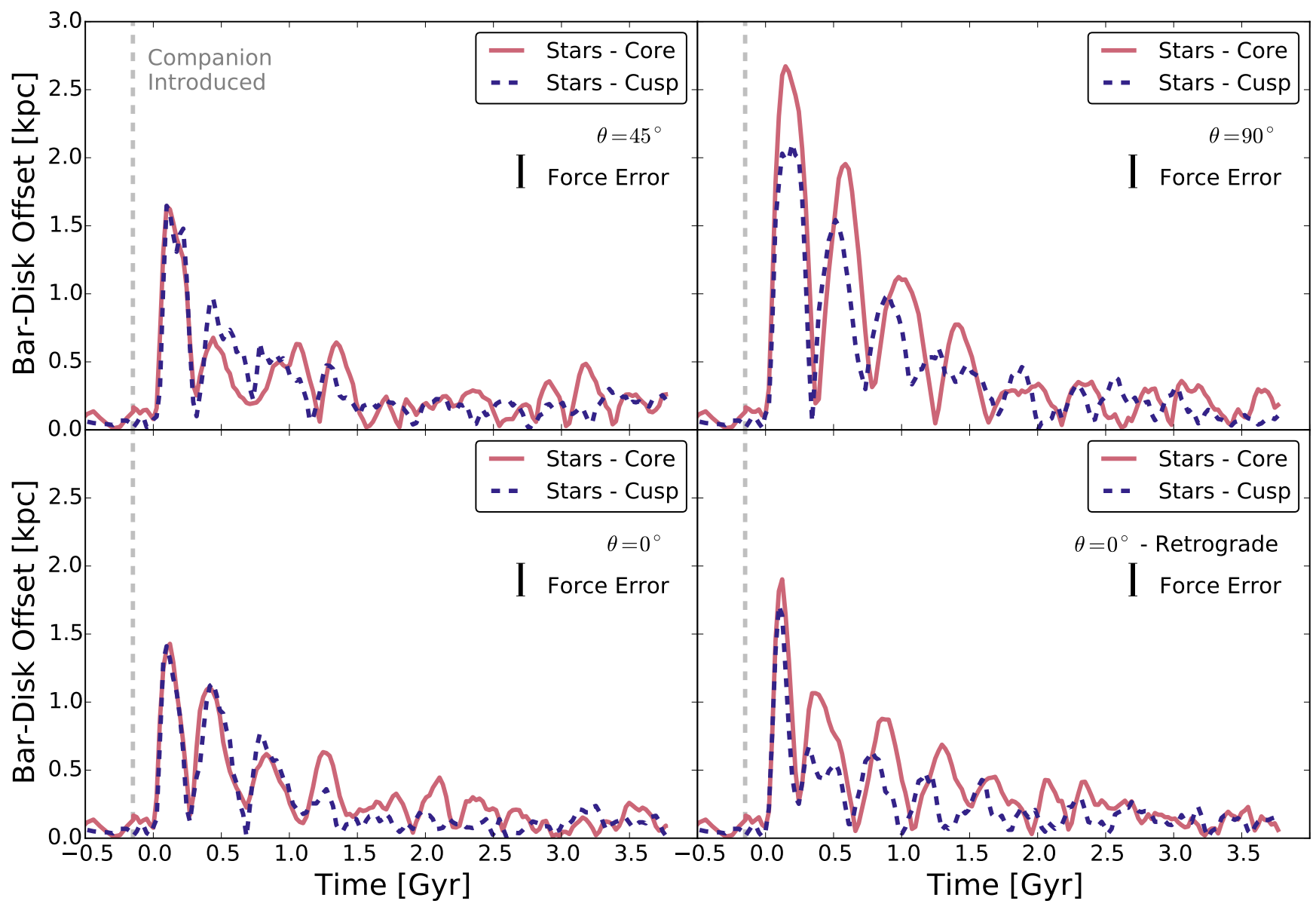

Figure 5. Time evolution of the displacement between the bar and disk centers shown for the same four orbital orientations as in Figure 3 and for both halo types. We compare models with a cored halo density profile (red lines) to models with a Hernquist inner-density profile for the dark halo (blue dashed-lines). The time of closest approach is set at $0 \mathrm{Gyr}$, and we include 0.5 Gyr of evolution before the introduction of a companion.

ing a 2D nonaxisymmetric disk model as implemented the code DiskFit (Spekkens \& Sellwood 2007, Sellwood \& Sánchez 2010). This method uses a nonaxisymmetric model to fit the kinematic data of the gas particles within a radius of $14 \mathrm{kpc}$ and compares with previous methods.

To further test how the asymmetries appear in the velocity distributions, we measure the average in-plane velocity of the disk stars in the each pixel of our surface density map. We demonstrate this method by superposing the velocity vectors on the surface density in Figure 7 . This method is similar to the one applied to the LMC stellar disk using proper-motion rotation fields by van der Marel \& Kallivayalil (2014).

The centers obtained with this method at each time are compared to the dynamical center as previously measured and displayed in Figure 8 . The stellar photometric center obtained by fitting ellipses to the stellar disk and bar is shown (solid black line), as compared to the the gas velocity field center measured by DiskFit (dotted blue line), and with the stellar velocity field center described above (solid brown line).

First, we notice that the velocity field center of the stellar disk is never coincident with the dynamical center, defined as the location of the deepest potential well, but it is always displaced on average $0.5 \mathrm{kpc}$, and with a maximum displacement of $\sim 1 \mathrm{kpc}$ when $\theta=90^{\circ}$.
Our findings are in agreement with Magellanic spiral galaxies observed in the field. In these systems the dynamical center, usually assumed to be traced from $\mathrm{HI}$ rotation curves, is coincident with the center of the bar and offset with respect to the photometric center of the stellar disk, as shown in the Magellanic galaxy NGC 4027 (Phookun et al. 1992) and in NGC 3906 (Watson et al. 2011; de Swardt et al. 2015). Similar behavior was found by Matthews \& Gallagher (2002) studying a sample of typical late-type spiral galaxies. In particular, the latter work showed that the global H I profiles were generally symmetric and traced the center of the galaxy's potential even when the optical image or kinematic data showed asymmetry.

We also observe that the center location found for the gas velocity field is different than the center location of the stellar disk as measured by the the outer ellipse. Minor offsets between the gas and stars, like the one we find here, have been seen before in spiral arms created during encounters with lower-mass companions (Pettitt et al. 2016) and along the leading edges of strong central bars (Prendergast 1983; Athanassoula 1992; Sheth et al. $2002)$.

\subsection{Determination of the $L M C$ dynamical center}

There have been some questions raised about whether the bar in the LMC is a real dynamical feature. Zaritsky 


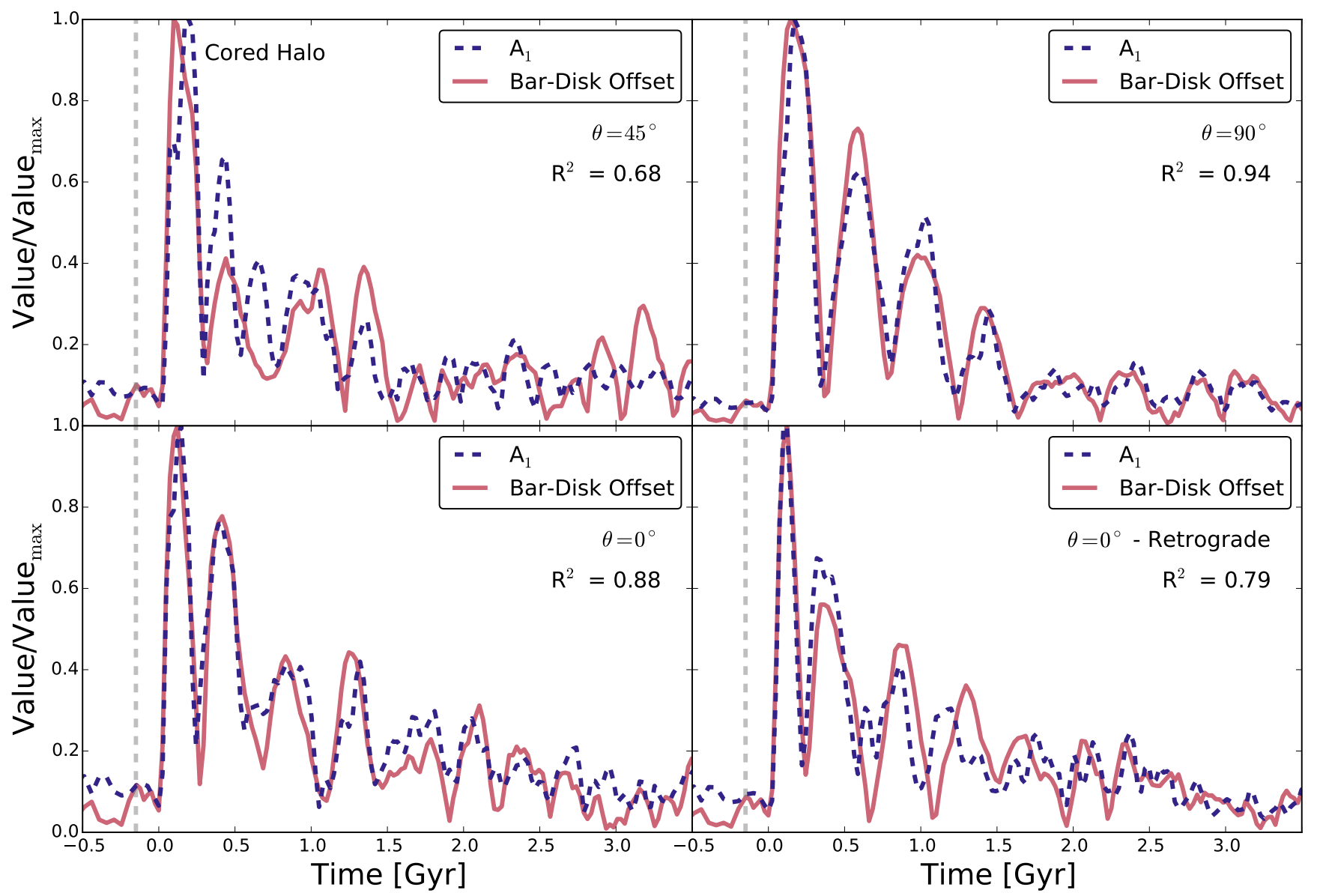

Figure 6. Time evolution of the asymmetry of the stellar disk in the cored halo compared with the bar-disk offset for the same four orbital orientations as in Figure 3 The two quantities have been normalized to their maximum value over the full time range. We show the $\mathrm{R}^{2}$ value in each panel, quantifying the degree to which the two time series coincide.

(2004) argues that it might be a triaxial bulge viewed edge-on, but Subramaniam \& Subramanian (2009) show that the bar is clearly part of the disk and may be influencing the gas (Indu \& Subramaniam 2015).

The bar is thought to be offset from the disk center (van der Marel 2001) and from the dynamical center as measured from H I kinematic tracers (Luks \& Rohlfs 1992 Kim et al. 1998, van der Marel 2001). Interestingly, the bar and outer disk are both consistent, within the estimated uncertainties, with the dynamical center as traced by the line-of-sight velocities of carbon stars (van der Marel et al. 2002).

Using stellar proper-motion fields, van der Marel \& Kallivayalil (2014) determined a more accurate dynamic center that is coincident with the H I center. According to our results such an offset can occur very soon after the encounter with a companion, as was likely the case with the LMC 200 Myr ago (Besla et al. 2012) when it collided with the SMC.

Following van der Marel \& Kallivayalil (2014) (their Fig. 2), we mapped the center of our simulated twodimensional stellar velocity map of the LMC analog primary galaxy after the collision with the SMC analog. In order to mock the observed stellar velocity map, we chose a sample of 22 fields (the number of fields used in the analysis of van der Marel \& Kallivayalil (2014)) within 5 $\mathrm{kpc}$ of the disk center of mass and fit the rotation curve:

$$
V(r)=V_{0} \times \min \left[\mathrm{r} / \mathrm{R}_{0}, 1\right],
$$

with $r=\sqrt{\left(x-x_{0}\right)^{2}+\left(y-y_{0}\right)^{2}}$. Here $\left(x_{0}, y_{0}\right)$ are center coordinates, $V_{0}$ is the maximum velocity, and $R_{0}$ is the turnover radius. We repeat this sampling process 10,000 times at each time step, mocking the process of observing a random population of stars of the galaxy as in the observations. We measure in this way at each time a systematic offset from the true dynamical center, traced in our simulations by the location where the potential is deepest. The displacement is amplified during the collision as shown in Figure 8 (solid brown line).

Our results predict that the halo center of the LMC is coincident with the bar center and that the H I centers will realign with this center over the next few hundred million years. If confirmed, this finding suggests that the bar center should be assumed as dynamical center instead of the H I center that has been previously used (Kallivayalil et al. 2013). This choice would increase the north component direction of the proper-motion measurements, $\mu_{N}$, from the current value $\mu_{N}=0.229 \pm 0.047$ to values close to the previous estimate of $\mu_{N}=0.34$ (van der Marel et al. 2002). The north component of the proper-motion of the LMC controls the location of the orbit when projected on the plane of the sky. This correction will increase the offset between the LMC's orbit and the position of the Magellanic Stream, an issue still 


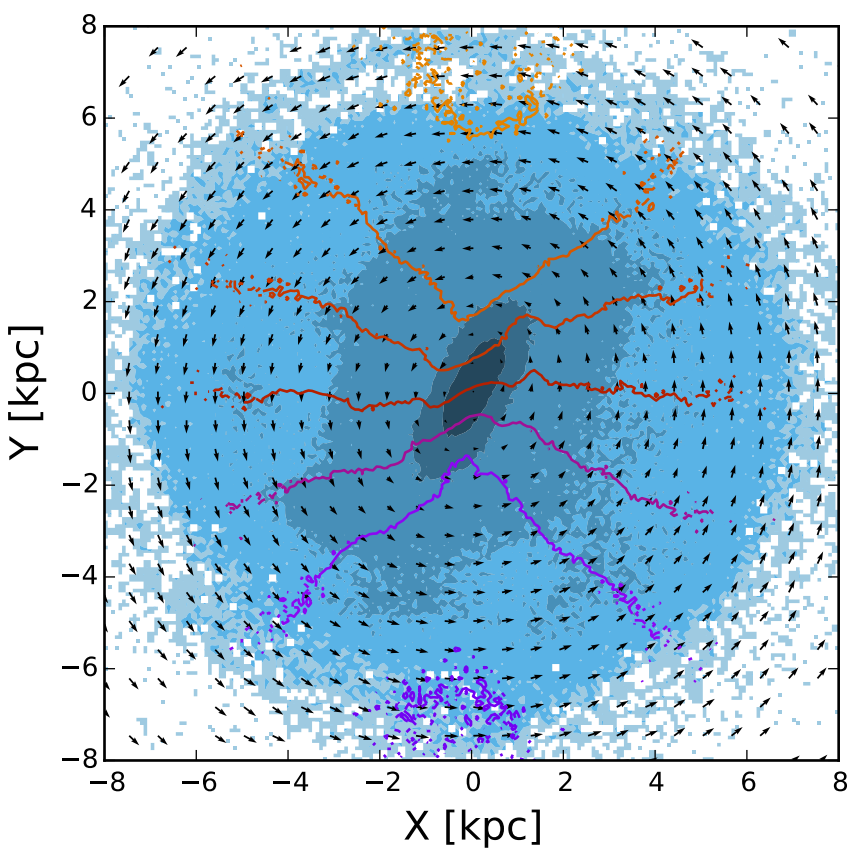

Figure 7. Velocity fields of the stellar and gas components overlaid on the stellar density distribution from the primary galaxy just before the encounter with the companion galaxy occurs. This model assumes a cored halo profile for the primary galaxy. The blue filled contours in the background display the stellar density distribution as in the first row of Figure 2 The colored contours show the gaseous velocity fields as on the bottom row of Figure 2 The arrows show the average stellar motion in the $x-y$ plane in each pixel. Note that only $1 / 8$ of the arrows are plotted here to improve clarity.

unsolved in the studies on the origin of the Stream.

\section{DISCUSSION AND CONCLUSIONS}

We have investigated the perturbations induced in the stellar and gas disc and the bar of a Magellanic-type spiral galaxy by a fly-by with a companion. A set of numerical experiments have been studied varying the angle between the orbital plane and the equatorial plane of the primary galaxy. The dynamical response of the disk has also been analyzed for a cored or Hernquist dark halo profile for the Magellanic-type galaxy. The results can be summarized as follows:

1. The bar center is always coincident with the dynamical center, suggesting that, contrary to common belief, the bar is never displaced in Magellanic spiral galaxies. Instead, the stellar disk is measured to be shifted from the dynamic center and the bar of the primary galaxy by, at most, 1.5-2.5 $\mathrm{kpc}$, depending on the details of the encounter with the companion. Thus, the observed displacements should, on average, be well below this value, in good agreement with observations (e.g. Feitzinger 1980). The disk distortions and asymmetries persist for almost 2 Gyr, the time that it takes for the disk to be recentered, and well after the interaction with the companion ended. The disk sloshing around the dynamical center reduces in amplitude with time.

2. Disk asymmetries are slightly more pronounced in
Magellanic galaxies with a cored halo profile as compared to models with a cuspy halo profile for the dark matter, but the differences are modest. The largest response of the disk of the primary galaxy tidally induced by the passage of the companion occurs for smaller impact parameters and depends on the orientation of the orbital plane with respect to the primary galaxy's equatorial plane.

3. The gas disk also sloshes similarly to the stellar disk during the gravitational interaction with the companion, but with a modest amplitude, and it tends to recenter after a short time due to the dissipative nature of the gas.

4. These results, when applied to the LMC - the prototype of the Magellanic spiral galaxies - suggest that the dynamical center should reside in the bar center instead of the $\mathrm{H}$ I center usually assumed in previous works. This choice would imply a change in the north component of the LMC proper-motion estimates, perhaps pointing to interesting implications for the offset between the LMC's orbit and the position of the Magellanic Stream. However, the little observational evidence of a bar in the $\mathrm{H}$ I gas distribution (Staveley-Smith et al. 2003) remains a challenge for theoretical models.

Our results might be of interest for interpreting the recent discoveries of bulgeless galaxies with off-center 'nuclear' clusters (Gallagher, J.S. 2016 private communication). This is a larger class of objects with stellar disks showing mismatched photometric centers. According to our results, their dynamical centers are likely to reside in the nuclear clusters, while the irregularities and asymmetries of the stellar disks should be interpreted as the outcome of tidally-induced distortions.

Support for this research was provided by the University of Wisconsin - Madison Office of the Vice Chancellor for Research and Graduate Education with funding from the Wisconsin Alumni Research Foundation. This research is also funded by NSF Grant No AST1211258 and ATP NASA Grant No NNX144AP53G. ED gratefully acknowledges the support of the Alfred P. Sloan Foundation. EA acknowledges financial support from the EU Programme FP7/2007-2013/, under REA grant PITN-GA-2011-289313 and from CNES (Centre National d'Etudes Spatiales, France). We express our appreciation to the Aspen Center for Physics for their hospitality, funded by the NSF under Grant No. PHYS-1066293. Simulations have been run on the High Performance Computing cluster provided by the Advanced Computing Infrastructure (ACI) and Center for High Throughput Computing (CHTC) at the University of Wisconsin. This research made use of Astropy ${ }^{2}$, a community-developed core Python package for Astronomy (Astropy Collaboration, 2013). This research made use of the Patho ${ }^{3}$ multiprocessing library $\mathrm{McK}-$ erns et al. 2012). This research has made use of the NASA/IPAC Extragalactic Database (NED), which is

\footnotetext{
2 http://www.astropy.org/

3 http://trac.mystic.cacr.caltech.edu/project/pathos
} 


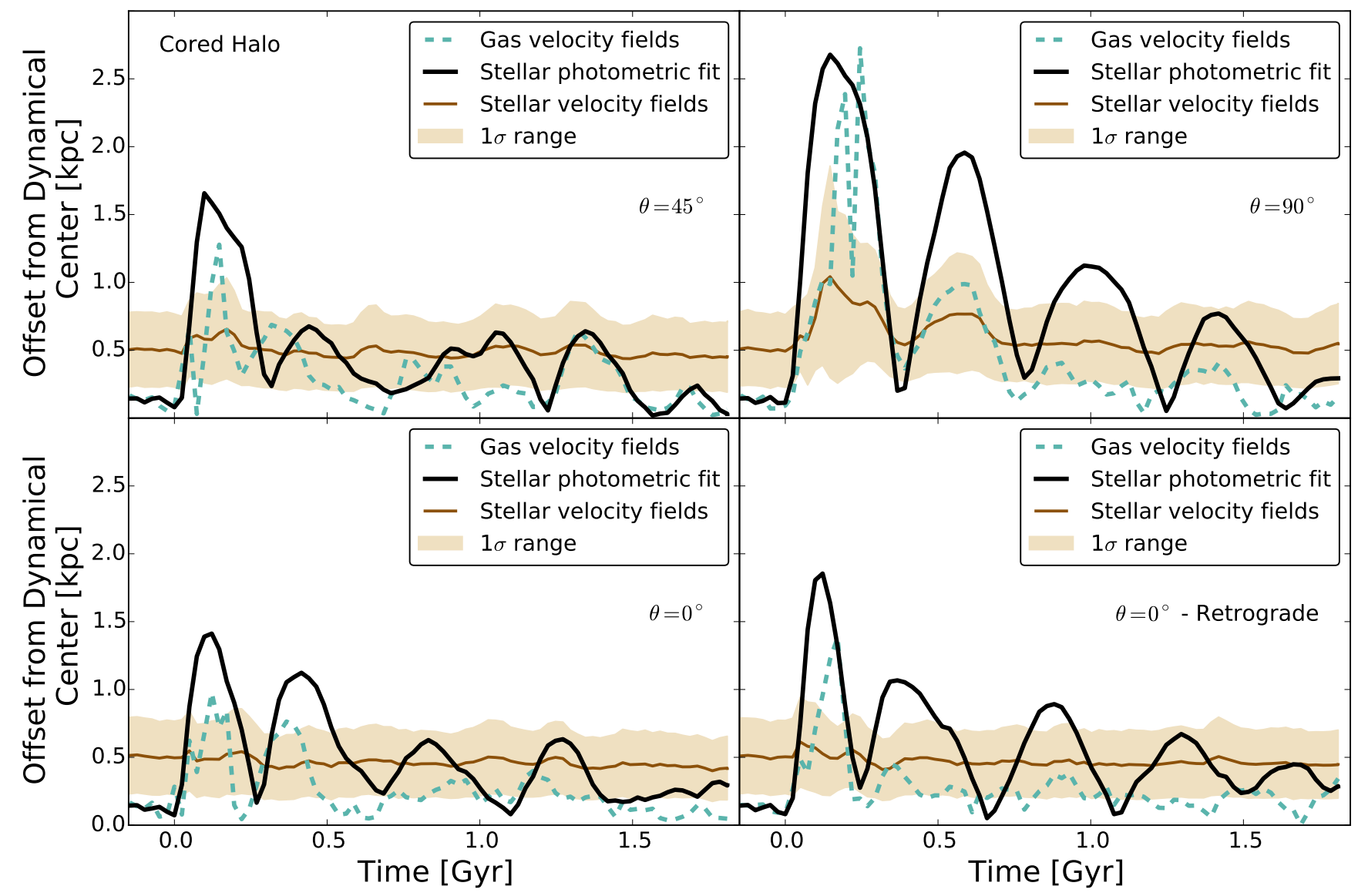

Figure 8. Time sequence of offset between gas and stellar disk and the dynamical center as measured by using different methods and for encounters with different orbital configurations. The primary galaxy has a cored halo profile. Centers of the disk measured from the photometric ellipse fitting are shown (thick black line). Centers of the gaseous disk measured using $2 \mathrm{D}$ tilted ring fitting of the gaseous velocity field by DiskFit are also displayed (dotted blue line) and compared to centers of the stellar disk as measured using the average in-plane velocity of the stars (thin brown line). The solid line shows the average of all 10,000 trials (see discussion in Section 3.5), and the filled region shows the standard deviation of all the trials.

operated by the Jet Propulsion Laboratory, California Institute of Technology, under contract with the National Aeronautics and Space Administration, and NASA's Astrophysics Data System.

\section{REFERENCES}

Athanassoula, E. 1992, MNRAS, 259, 345

-. 1996, Barred galaxies. Astronomical Society of the Pacific Conference Series, 91, 309

Athanassoula, E., Puerari, I., \& Bosma, A. 1997, MNRAS, 286, 284

Bekki, K. 2009, MNRAS Letters, 393, L60

Berentzen, I., Athanassoula, E., Heller, C. H., \& Fricke, K. J. 2003, MNRAS, 341, 343

Besla, G., Kallivayalil, N., Hernquist, L., et al. 2010, ApJ, 721, L97

-. 2012, MNRAS, 421, 2109

Bournaud, F., Combes, F., Jog, C. J., \& Puerari, I. 2005, A\&A, 438, 507

Buta, R. J., Sheth, K., Athanassoula, E., et al. 2015, ApJS, 217, 32

Colin, J., \& Athanassoula, E. 1989, A\&A, 214, 99

de Blok, W. J. G., McGaugh, S. S., Bosma, A., \& Rubin, V. C. 2001, ApJ, 552, L23

de Swardt, B., Sheth, K., Kim, T., et al. 2015, ApJ, 808, 90

de Vaucouleurs, G. 1963, ApJS, 8, 31

de Vaucouleurs, G., \& Freeman, K. C. 1972, Vistas in Astronomy, 14, 163

Dehnen, W. 1993, MNRAS, 265, 250
D'Onghia, E. 2015, ApJ, 808, L8

D’Onghia, E., Besla, G., Cox, T. J., \& Hernquist, L. 2009, Nature, 460, 605

D’Onghia, E., \& Fox, A. J. 2015, eprint arXiv:1511.05853, 1511.05853

D’Onghia, E., Vogelsberger, M., Faucher-Giguere, C.-A., \& Hernquist, L. 2010, ApJ, 725, 353

Eskridge, P. B., Frogel, J. A., Pogge, R. W., et al. 2000, AJ, 119, 536

Feitzinger, J. V. 1980, Space Science Reviews, 27, 35

Gajda, G., \& Lokas, E. L. 2015, eprint arXiv:1508.03149, 1508.03149

Hernquist, L. 1990, ApJ, 356, 359

Indu, G., \& Subramaniam, A. 2015, A\&A, 573, A136

Kallivayalil, N., van der Marel, R. P., Besla, G., Anderson, J., \& Alcock, C. 2013, ApJ, 764, 161

Kim, S., Staveley-Smith, L., Dopita, M. A., et al. 1998, ApJ, 503, 674

Łokas, E. L., Athanassoula, E., Debattista, V. P., et al. 2014, MNRAS, 445, 1339

Łokas, E. L., Semczuk, M., Gajda, G., \& D’Onghia, E. 2015, ApJ, 810,100

Luks, T., \& Rohlfs, K. 1992, A\&A, 263, 41

Mapelli, M., Moore, B., \& Bland-Hawthorn, J. 2008, MNRAS, 388,697

Marinova, I., \& Jogee, S. 2007, ApJ, 659, 1176

Matthews, L. D., \& Gallagher, J. S. I. 2002, ApJS, 141, 429 
McGaugh, S. S. 2002, in THE SHAPES OF GALAXIES AND THEIR DARK HALOS. Proceedings of the Yale Cosmology Workshop. Held 28-30 May 2001 in New Haven, Department of Astronomy, University of Maryland, College Park, MD, USA ssm@astro.umd.edu, 186-193

McKerns, M. M., Strand, L., Sullivan, T., Fang, A., \& Aivazis, M. A. G. $2012,1202.1056$

Menendez-Delmestre, K., Sheth, K., Schinnerer, E., Jarrett, T. H., \& Scoville, N. Z. 2007, ApJ, 657, 790

Muñoz-Mateos, J. C., Sheth, K., Gil de Paz, A., et al. 2013, ApJ, 771,59

Odewahn, S. C. 1991, AJ, 101, 829

Oh, S.-H., Brook, C., Governato, F., et al. 2011, AJ, 142, 24

Oman, K. A., Navarro, J. F., Fattahi, A., et al. 2015, MNRAS, 452, 3650

Pettitt, A. R., Tasker, E. J., \& Wadsley, J. W. 2016, MNRAS, 458, 3990

Phookun, B., Mundy, L. G., Teuben, P. J., \& Wainscoat, R. J. 1992, ApJ, 400, 516

Prendergast, K. H. 1983, in IN: Internal kinematics and dynamics of galaxies; Proceedings of the Symposium, Columbia University, New York, NY, 215-220

Rodionov, S. A., Athanassoula, E., \& Sotnikova, N. Y. 2009, MNRAS, 392, 904

Ross, A. 2012, in American Astronomical Society, 417.06

Sellwood, J. A., \& Sánchez, R. Z. 2010, MNRAS, 404, 1733

Sheth, K., Vogel, S. N., Regan, M. W., et al. 2002, AJ, 124, 2581
Sheth, K., Elmegreen, D. M., Elmegreen, B. G., et al. 2008, ApJ, 675,1141

Sheth, K., Regan, M., Hinz, J. L., et al. 2010, PASP, 122, 1397

Spekkens, K., \& Sellwood, J. A. 2007, ApJ, 664, 204

Springel, V. 2005, MNRAS, 364, 1105

Springel, V., Di Matteo, T., \& Hernquist, L. 2005, MNRAS, 361, 776

Springel, V., \& Hernquist, L. 2002, MNRAS, 333, 649

-. 2003, MNRAS, 339, 289

Staveley-Smith, L., Kim, S., Calabretta, M. R., Haynes, R. F., \& Kesteven, M. J. 2003, MNRAS, 339, 87

Subramaniam, A., \& Subramanian, S. 2009, ApJ, 703, L37

Udalski, A., Szymanski, M. K., Soszynski, I., \& Poleski, R. 2008, Acta Astronomica, 58, 69

van der Marel, R. P. 2001, AJ, 122, 1827

van der Marel, R. P., Alves, D. R., Hardy, E., \& Suntzeff, N. B. 2002, AJ, 124, 2639

van der Marel, R. P., \& Kallivayalil, N. 2014, ApJ, 781, 121

Watson, L. C., Schinnerer, E., Martini, P., Böker, T., \& Lisenfeld,

U. 2011, The Astrophysical Journal Supplement, 194, 36

Wilcots, E. M., \& Prescott, M. K. M. 2004, AJ, 127, 1900

Yozin, C., \& Bekki, K. 2014, MNRAS, 439, 1948

Yurin, D., \& Springel, V. 2014, MNRAS, 444, 62

Zaritsky, D. 2004, ApJ, 614, L37

Zaritsky, D., \& Rix, H.-W. 1997, ApJ, 477, 118

Zaritsky, D., Salo, H., Laurikainen, E., et al. 2013, ApJ, 772, 135

Zhao, H., \& Evans, N. W. 2000, ApJ, 545, L35 